\title{
Microdeletion on 17p11.2 in a Smith-Magenis syndrome patient with mental retardation and congenital heart defect: first report from China
}

\author{
C. Huang ${ }^{1}$, Y.-F. Yang ${ }^{1,2}$, H. Zhang, ${ }^{1}$ L. Xie ${ }^{1}$, J.-L. Chen ${ }^{1}$, J. Wang ${ }^{2}$, \\ Z.-P. Tan ${ }^{1,2}$ and H. Luo ${ }^{2,3}$ \\ 'Department of Cardiothoracic Surgery, Second Xiangya Hospital, \\ Central South University, Changsha, Hunan, China \\ ${ }^{2}$ State Key Laboratory of Medical Genetics, \\ Clinical Center for Gene Diagnosis and Therapy, Second Xiangya Hospital, \\ Central South University, Changsha, Hunan, China \\ ${ }^{3}$ Department of Respiratory Medicine, Second Xiangya Hospital, \\ Central South University, Changsha, Hunan, China \\ Corresponding author: H. Luo \\ E-mail: luohong1003@163.com
}

Genet. Mol. Res. 11 (3): 2321-2327 (2012)

Received February 1, 2012

Accepted July 6, 2012

Published August 13, 2012

DOI http://dx.doi.org/10.4238/2012.August.13.5

\begin{abstract}
Smith-Magenis syndrome (SMS) is a rare syndrome with multiple congenital malformations, including development and mental retardation, behavioral problems and a distinct facial appearance. SMS is caused by haploinsufficiency of RAII (deletion or mutation of RAII). We describe an eight-year-old female Chinese patient with multiple malformations, congenital heart defect, mental retardation, and behavioral problems (self hugging, sleeping disturbance). High-resolution genome wide single nucleotide polymorphism array revealed a $3.7-\mathrm{Mb}$ deletion in chromosome region $17 \mathrm{p} 11.2$. This chromosome region contains $R A I 1$, a critical gene involved in SMS. To
\end{abstract}


the best of our knowledge, this is the first report of an SMS patient in mainland China.

Key words: Smith-Magenis syndrome; Congenital heart defect; High-resolution single nucleotide polymorphism;

Sleeping disturbance; Mental retardation

\section{INTRODUCTION}

Smith-Magenis syndrome (SMS; OMIM\#182290) is a rare kind of syndrome with multiple congenital malformations, including developmental and mental retardation (MR), behavioral problems, sleeping disturbance, and distinct facial appearance (Stratton et al., 1986). Since two unrelated patients with facial clefts and congenital heart defects (CHD) were first reported by Smith in 1982, various clinical phenotypes have been described in patients with interstitial deletion of 17p11.2 (Smith et al., 1986), and subsequently defined as a deletion syndrome in 1986 by Stratton et al. This syndrome is clinically diagnosed by the distinct facial features in combination with behavioral problems. SMS is caused by haploinsufficiency of RAIl, either by $17 \mathrm{p} 11.2$ microdeletions containing the $R A I 1$ gene or mutations within this gene (Greenberg et al., 1991; Slager et al., 2003). The incidence of this syndrome has been estimated to be 1:15,000 25,000 births (Greenberg et al., 1991). However, due to the insufficient sensitivity of traditional techniques, microdeletions of SMS are often underdiagnosed (Greenberg et al., 1991). Recent developments of single nucleotide polymorphism (SNP) array allow for genome-wide screening at a resolution that is undetectable (smaller than $5 \mathrm{Mb}$ in size) by traditional cytogenetic methods and have facilitated the discovery of a number of novel microdeletion and microduplication syndromes (Slavotinek, 2008).

As part of a larger study on the identification of pathogenic copy number variations in children with CHD (Tan et al., 2011; Chen et al., 2012; Huang et al., 2012), high-resolution SNP array (Illumina, San Diego, CA, USA) has been performed in CHD children and their parents. Here, we present a detailed study of an 8-year-old female Chinese patient with facial dysmorphisms, behavioral problems, CHD, and MR.

\section{MATERIAL AND METHODS}

The Review Board of the Second Xiangya Hospital of the Central South University approved this research. All subjects consented to this study.

\section{Clinical presentation}

In 2010, an 8-year-old female from Central-South China was seen at our hospital for a heart murmur. She was the second child of unrelated parents and had no family history of inherited diseases. She was born at 37 weeks with a birth weight of $3.2 \mathrm{~kg}(50 \mathrm{th})$ and a length of $42 \mathrm{~cm}(<3 \mathrm{rd})$. At birth, she presented lip cyanosis, clubbing and heart defects (tetralogy of Fallot, atrium septum defect and patent duct artery). At 2 years old, behavior problems such as self-injury, toilet difficulties and fragmented sleep cycles with frequent nocturnal awakenings gradually appeared. At the last examination ( 8 years old), she had a height of $110 \mathrm{~cm}(<3 \mathrm{rd})$ 
with a weight of $16.5 \mathrm{~kg}(<3 \mathrm{rd})$. A moderate mental retardation and a decreased ability were observed, and her IQ was 49 compared to 80 at two years old. She had hearing loss and speech problems, she only spoke some single words such as ma and pa. Additionally, she had a distinct facial appearance of a round face with depressed nasal bridge, hypertelorism, an everted upper lip with a "tented" appearance shaped like a "V", and tooth agenesis (Figure 1).

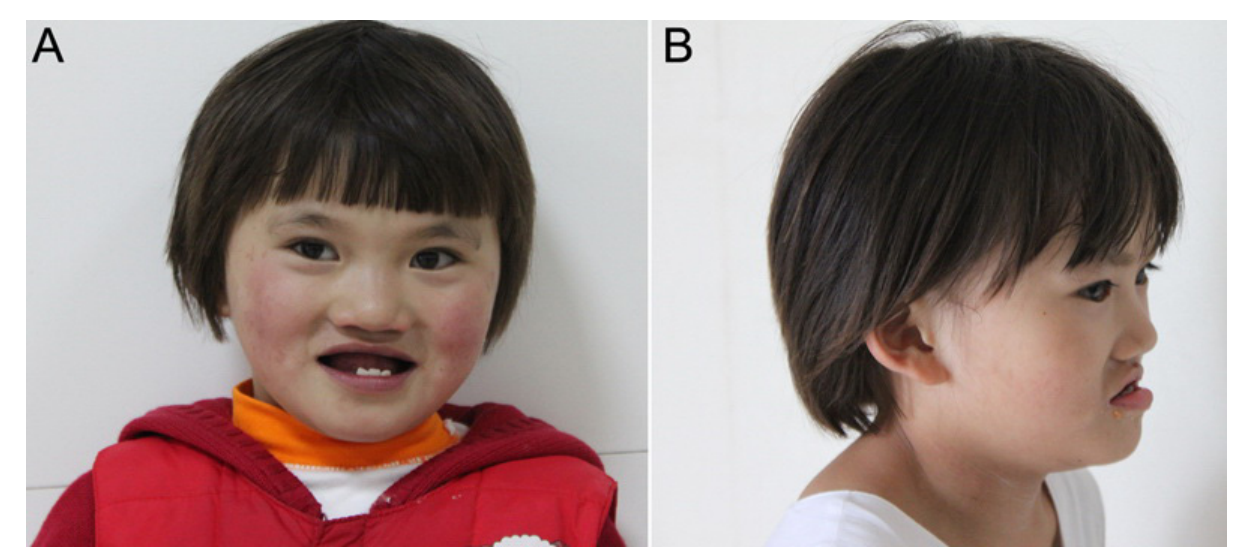

Figure 1. Distinct facial features of the proband, the patient has a round face with depressed nasal bridge, hypertelorism, an everted upper lip with a "tented" appearance like the character "V", and tooth agenesis. Frontal (A) and lateral (B) view of the patient.

\section{Cytogenetic analysis}

Chromosome analysis was performed with the patient's and her parents' peripheral blood samples by conventional G-banding techniques (550-band resolution). A sample of 2 $\mathrm{mL}$ peripheral blood was collected. All samples were subjected to lymphocyte culture according to standard cytogenetic protocol.

\section{SNP array analysis}

Following informed consent, peripheral venous blood was collected. Genomic DNA was isolated from peripheral blood leukocytes using a QIAamp DNA Blood Mini kit (Qiagen, Valencia, CA, USA) according to manufacturer instructions and was adjusted to a final concentration $(100 \mathrm{ng} / \mu \mathrm{L})$. The Human660w-Quad Chip (Illumina Inc.) and the Illumina BeadScan genotyping system (Beadstation Scanner) were employed to obtain the signal intensities of probes (SNP) as previously described (Huang et al., 2012).

\section{RESULTS}

The chromosome analysis of the patient revealed a normal female karyotype, described as 46,XX. Her parents also had a normal karyotype. Clinical examination showed a combination of phenotypes with CHD, MR, facial features, and behavior issues, raising con- 
cerns about a chromosomal abnormality of microdeletion/microduplication. To explore the exact genomic lesion of this patient, we employed the SNP array system (Human660w-Quad Chip, Beadstation Scanner and BeadStudio 3.3.7 software) to analyze the whole genome copy number variations. Comparing with the Database of Genomic Variants (DGV), we identified a de novo 3.7-Mb deletion at chromosome 17p11.2 (Chr17: 16720137-20378904) (NCBI build 37/Hg19). This chromosome region contains about 50 annotated genes, including RAI1, MYO15A, AKAP10, and ALDH3A2 (Figure 2). Her parents did not carry this genomic lesion.

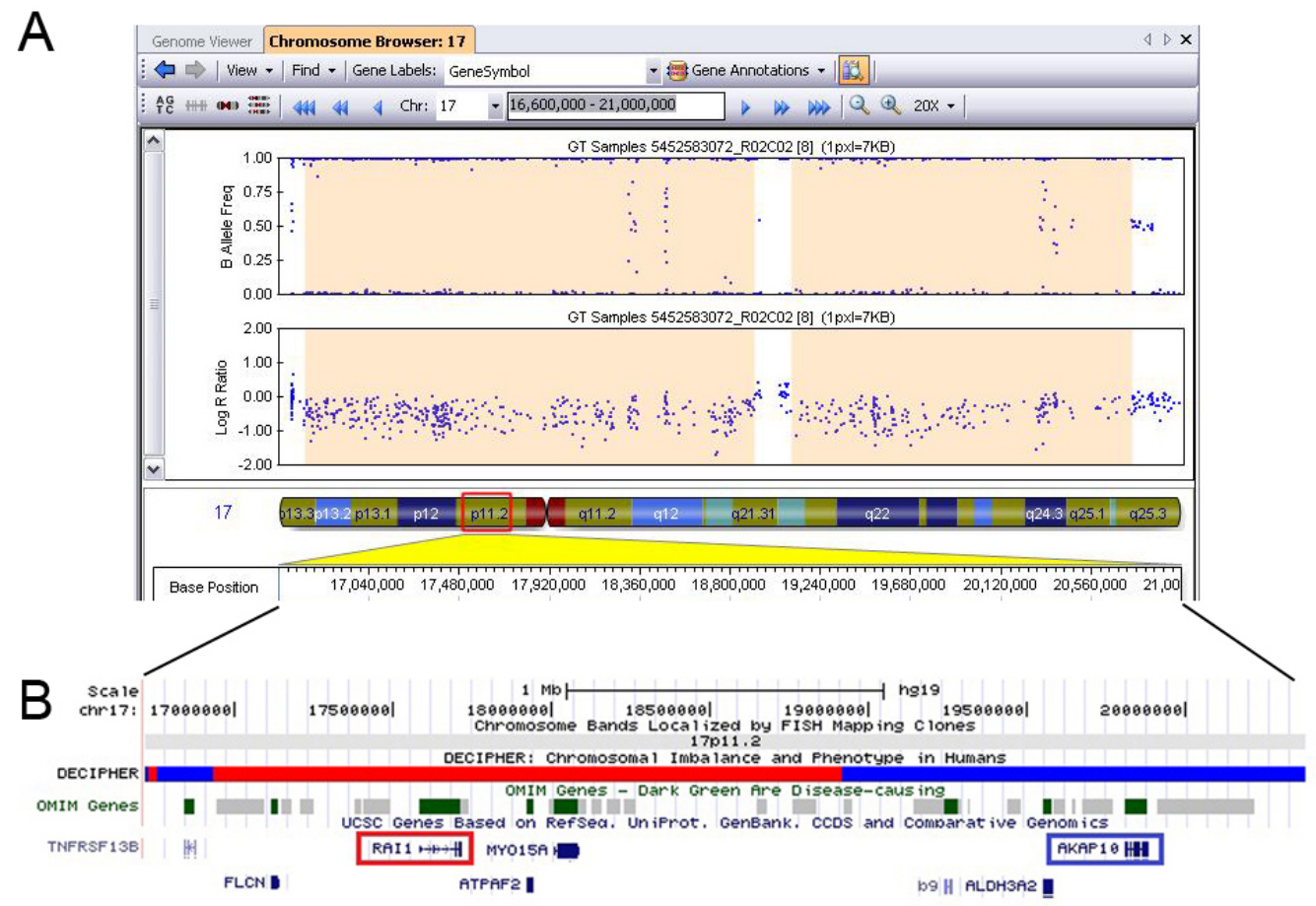

Figure 2. Human $660 \mathrm{w}-$ Quad SNP array result of $17 \mathrm{p} 11.2$ deletion in the proband. The above panel shows the region involved in cytogenetic bands $17 \mathrm{p} 11.2$. A de novo $3.7-\mathrm{Mb}$ deletion on chromosome 17p11.2 (Chr17: 16720137-20378904) is identified (UCSC Genome Browser on Human GRCh37/hg19 Assembly). The lower panel shows the key annotated genes. AKAP10 might be an interesting candidate gene for CHD.

\section{DISCUSSION}

We report here on an 8-year-old female with a de novo microdeletion of $3.7 \mathrm{Mb}$ in 17p11.2. She had normal karyotype and presented clinical SMS-like syndrome phenotypes comprising several malformations, MR, CHD (tetralogy of Fallot), marked sleeping disturbance, and behavioral problems. By recent SNP array technology, this patient was finally diagnosed with SMS. To the best of our knowledge, she is the first reported SMS patient in China (mainland). 
The phenotypes of SMS are characterized by neurological, developmental and behavioral problems (Elsea and Girirajan, 2008). While a wide phenotypic variability is observed in SMS patients, some patients show mild to moderate mental retardation, and some patients may not have typical facial features, cardiac, renal, and otolaryngologic abnormalities (Edelman et al., 2007). In developing countries, molecular approaches such as fluorescence in situ hybridization and microarray are limited (Gamba et al., 2011), and diagnosis is largely based on clinical recognition of the combination of these features, making accurate assessment and diagnosis of SMS difficult in children (Girirajan et al., 2006; Gropman et al., 2006). Another explanation for the difficulty in diagnosis is that SMS shares phenotypic features with other syndromes (Elsea and Girirajan, 2008). Sleep disturbance, MR, flat face, and cardiac defect are clinical suspicions in both SMS and 9q34 deletion syndrome (Kleefstra et al., 2009). Also, hearing loss, eating difficulties and MR are also frequently present in 22q11.2 deletion syndrome (Kobrynski and Sullivan, 2007). SMS also shares self-injurious, obsessive-compulsive behaviors and short stature with Prader-Willi syndrome (Cassidy and Driscoll, 2009). One additional explanation for the first report of SMS in China is that parents do not care about the molecular diagnosis of neurological diseases, especially those in the suburban and rural region of China (Shi and Jia, 2011).

Our patient showed a complex CHD (tetralogy of Fallot, atrium septum defect and patent duct artery). CHD are frequently present in the SMS patient (with an incidence of 37\%) (Greenberg et al., 1996), including the first two patients reported in 1982 (Smith et al., 1982). Tetralogy of Fallot is rare and few patients have been described in previous reports (Sweeney et al., 1999; Wong et al., 2003). Other CHD such as ventricular/atrium septum defect, mitral valve prolapse/regurgitation, tricuspid valve stenosis/regurgitation, supravalvular pulmonary stenosis, and subvalvular aortic stenosis have also been reported to be associated with SMS (Greenberg et al., 1996; Myers and Challman, 2004).

The discordance between phenotypes of $17 \mathrm{p} 11.2$ deletion (containing the RAII gene) and mutations within RAII highlights the contribution of other gene(s) to some specific phenotypes of SMS (CHD, renal anomalies) (Girirajan et al., 2005; Elsea and Girirajan, 2008). Our findings also raise the question of related gene(s) for CHD in the $17 \mathrm{p} 11.2$ region. Fifty annotated genes, including RAI1, MYO15A, AKAP10, and $A L D H 3 A 2$ are found in this deletion region. AKAP10, a kinase anchor protein highly enriched in mitochondria, has been shown to modulate the sensitivity of cardiac pacemaker cells to sympathetic-vagal interactions at the sinus node in humans with cardiovascular diseases (Neumann et al., 2009). Mutations or deletions of $A L D H 3 A 2$, which encode an enzyme that catalyzes the oxidation of fatty aldehyde to fatty acid, have been shown to cause an autosomal recessive disorder Sjogren-Larsson syndrome (Engelstad et al., 2011). MYO15A has been identified to be responsible for deafness (Kalay et al., 2007). However, due to the lack of molecular data and small sample size in previous studies, the precise gene(s) responsible for CHD in SMS is still unclear.

Given that SMS may not be well known to clinical cardiologists, our study will help the clinical recognition of SMS in China. Our study with high-resolution SNP array data and detailed phenotypes also provides one more piece of evidence for the identification of causative gene(s) for CHD in SMS. With the increased number of SMS cases diagnosed by recent molecular approaches, the causative gene for CHD in SMS should be uncovered in the near future, and hopefully our study may help with early diagnosis, genetic counseling and effective long-term management of SMS. 


\section{ACKNOWLEDGMENTS}

Research supported by the National Natural Science Foundation of China (\#81101475). We thank all patients and their parents for participating in this study. We also thank the State Key Laboratory of Medical Genetics of China for technical assistance.

\section{Conflict of interest statement}

The authors declare no conflict of interest.

\section{REFERENCES}

Cassidy SB and Driscoll DJ (2009). Prader-Willi syndrome. Eur. J. Hum. Genet. 17: 3-13.

Chen JL, Yang YF, Huang C, Wang J, et al. (2012). Clinical and molecular delineation of 16p13.3 duplication in a patient with congenital heart defect and multiple congenital anomalies. Am. J. Med. Genet. A 158A: 685-688.

Edelman EA, Girirajan S, Finucane B, Patel PI, et al. (2007). Gender, genotype, and phenotype differences in SmithMagenis syndrome: a meta-analysis of 105 cases. Clin. Genet. 71: 540-550.

Elsea SH and Girirajan S (2008). Smith-Magenis syndrome. Eur. J. Hum. Genet. 16: 412-421.

Engelstad H, Carney G, S'aulis D, Rise J, et al. (2011). Large contiguous gene deletions in Sjogren-Larsson syndrome. Mol. Genet. Metab. 104: 356-361.

Gamba BF, Vieira GH, Souza DH, Monteiro FF, et al. (2011). Smith-Magenis syndrome: clinical evaluation in seven Brazilian patients. Genet. Mol. Res. 10: 2664-2670.

Girirajan S, Elsas LJ, Devriendt K and Elsea SH (2005). RAI1 variations in Smith-Magenis syndrome patients without 17p11.2 deletions. J. Med. Genet. 42: 820-828.

Girirajan S, Vlangos CN, Szomju BB, Edelman E, et al. (2006). Genotype-phenotype correlation in Smith-Magenis syndrome: evidence that multiple genes in 17p11.2 contribute to the clinical spectrum. Genet. Med. 8: 417-427.

Greenberg F, Guzzetta V, Montes dO-L, Magenis RE, et al. (1991). Molecular analysis of the Smith-Magenis syndrome: a possible contiguous-gene syndrome associated with del(17)(p11.2). Am. J. Hum. Genet. 49: 1207-1218.

Greenberg F, Lewis RA, Potocki L, Glaze D, et al. (1996). Multi-disciplinary clinical study of Smith-Magenis syndrome (deletion 17p11.2). Am. J. Med. Genet. 62: 247-254.

Gropman AL, Duncan WC and Smith AC (2006). Neurologic and developmental features of the Smith-Magenis syndrome (del 17p11.2). Pediatr. Neurol. 34: 337-350.

Huang C, Yang YF, Yin N, Chen JL, et al. (2012). Congenital heart defect and mental retardation in a patient with a 13q33.1-34 deletion. Gene 498: 308-310.

Kalay E, Uzumcu A, Krieger E, Caylan R, et al. (2007). MYO15A (DFNB3) mutations in Turkish hearing loss families and functional modeling of a novel motor domain mutation. Am. J. Med. Genet. A 143A: 2382-2389.

Kleefstra T, van Zelst-Stams WA, Nillesen WM, Cormier-Daire V, et al. (2009). Further clinical and molecular delineation of the 9q subtelomeric deletion syndrome supports a major contribution of EHMT1 haploinsufficiency to the core phenotype. J. Med. Genet. 46: 598-606.

Kobrynski LJ and Sullivan KE (2007). Velocardiofacial syndrome, DiGeorge syndrome: the chromosome 22q11.2 deletion syndromes. Lancet 370: 1443-1452.

Myers SM and Challman TD (2004). Congenital heart defects associated with Smith-Magenis syndrome: two cases of total anomalous pulmonary venous return. Am. J. Med. Genet. A 131: 99-100.

Neumann SA, Tingley WG, Conklin BR, Shrader CJ, et al. (2009). AKAP10 (I646V) functional polymorphism predicts heart rate and heart rate variability in apparently healthy, middle-aged European-Americans. Psychophysiology 46: 466-472.

Shi FD and Jia JP (2011). Neurology and neurologic practice in China. Neurology 77: 1986-1992.

Slager RE, Newton TL, Vlangos CN, Finucane B, et al. (2003). Mutations in RAI1 associated with Smith-Magenis syndrome. Nat. Genet. 33: 466-468.

Slavotinek AM (2008). Novel microdeletion syndromes detected by chromosome microarrays. Hum. Genet. 124: 1-17.

Smith AC, McGavran L, Waldstein G and Robinson J (1982). Deletion of the 17 short arm in two patients with facial clefts. Am. J. Hum. Genet. 34 (Suppl): 410A.

Smith AC, McGavran L, Robinson J, Waldstein G, et al. (1986). Interstitial deletion of (17)(p11.2p11.2) in nine patients. 
Am. J. Med. Genet. 24: 393-414.

Stratton RF, Dobyns WB, Greenberg F, DeSana JB, et al. (1986). Interstitial deletion of (17)(p11.2p11.2): report of six additional patients with a new chromosome deletion syndrome. Am. J. Med. Genet. 24: 421-432.

Sweeney E, Peart I, Tofeig M and Kerr B (1999). Smith-Magenis syndrome and tetralogy of Fallot. J. Med. Genet. 36: 501-502.

Tan ZP, Huang C, Xu ZB, Yang JF, et al. (2011). Novel ZFPM2/FOG2 variants in patients with double outlet right ventricle. Clin. Genet. DOI 10.1111/j.1399-0004.2011.01787.x.

Wong JT, Chan DK, Wong KY, Tan M, et al. (2003). Smith-Magenis syndrome and cyanotic congenital heart disease: a case report. Clin. Dysmorphol. 12: 73-74. 